\title{
Impacto de los diferentes métodos de pago para centros de atención ambulatoria
}

\author{
Impact of different payment methods for outpatient care facilities
}

Yuan B, y col. Cochrane Database of Systematic Reviews 2017, Issue 3. Art. No.: CD011153.

Objetivos

Evaluar el impacto de los diferentes métodos de pago en el rendimiento de los centros de atención ambulatoria y analizar las diferencias de efecto en escenarios diversos.

\section{Fuente de datos}

Se realizaron búsquedas en el Registro Cochrane CENTRAL de Ensayos Controlados; MEDLINE, OvidSP, Embase, PubMed, ProQuest; Conference Proceedings Citation Index (ISI Web of Science), IDEAS, EconLit, POPLINE, K4Health, Infraestructura Nacional del Conocimiento de China, Premier de medicina china, OpenGrey, ClinicalTrials.gov, Institutos Nacionales de Salud de Ios EE. UU. (NIH), Plataforma Internacional de Registro de Ensayos Clínicos (ICTRP) de la Organización Mundial de la Salud (OMS) y el sitio web del Banco Mundial (todos buscados el 8 de marzo de 2016) y OvidSP (buscado el 24 de abril de 2014). Además se revisaron las listas de referencias y búsqueda de citas para los estudios incluidos a través de ISI Web of Science para encontrar otros estudios potencialmente relevantes.

\section{Selección de estudios}

Se incluyeron ensayos aleatorizados, no aleatorizado, series de tiempo interrumpido, estudios de medidas repetidas, estudios controlados tipo antes y después, que compararon diferentes métodos de pago (presupuestos globales, presupuestos de partidas, capitación, pago por servicio, pago por desempeño y pago mixto), para centros de salud de atención ambulatoria que proporcionaron servicios de salud a personas que no requirieron hospitalización o institucionalización. Solo se incluyeron métodos utilizados para transferir fondos del comprador de servicios de salud a instalaciones de salud (incluidos grupos de profesionales individuales). Los resultados primarios fueron cantidad y calidad de los servicios brindados, utilización por parte de los pacientes de los servicios y resultados en la salud de los mismos, costos para los proveedores y efectos adversos. Dos investigadores independientes extrajeron los datos y evaluaron el riesgo de sesgo.

\section{Resultados principales}

Se incluyeron en la revisión 21 estudios de los 55.558 registros únicos encontrados luego de realizar la búsqueda. Los resultados se dividieron en tres tipos de comparaciones entre las formas de pago: -Pago por rendimiento (P4P) combinado con algún método de pago existente y los resultados del paciente. Los resultados se resumen en la tabla 1.b-Pago per cápita con pago por rendimiento comparado pago por atención brindada: se encontró un estudio llevado a cabo en China en el que se observa que el pago por rendimiento sumado al pago per cápita puede llevar a una ligera reducción en la prescripción de antibióticos (riesgo relativo 0,84; IC95\% 0,74 a 0,96; GRADE calidad moderada) en comparación al pago por atención brindada. No se hallaron diferencias en gastos, volumen de visitas o satisfacción de los pacientes. -Pago per cápita comparado pago por atención brindada: dos estudios de Estados Unidos llevados a cabo en centros de salud mental cuyo resultados fueron inciertos (GRADE calidad muy baja).

Tabla 1. Pago por rendimiento (P4P) combinado con algún método de pago existente y los resultados del paciente.

\begin{tabular}{|c|c|c|}
\hline Resultados & $\begin{array}{l}\text { RR ajustado para resultados dicotómicos } \\
\text { y mediana de cambio porcentual ajustada para resultados continuos (IC 95\%) }\end{array}$ & Calidad de evidencia (GRADE) \\
\hline $\begin{array}{l}\text { Resultados dicotómicos } \\
\text { (prescripción de pruebas o tratamiento) }\end{array}$ & RR ajustado $=1,095(1,01$ a 1,17) & $\begin{array}{l}\text { MODERADA } \\
\oplus \oplus \oplus \ominus\end{array}$ \\
\hline $\begin{array}{l}\text { Resultados continuos } \\
\text { (cumplimiento de criterios de calidad) }\end{array}$ & Mediana de cambio porcentual ajustada $=1,345 \%(8,49$ a 5,8$)$ & $\begin{array}{l}\text { MODERADA } \\
\oplus \oplus \oplus \ominus\end{array}$ \\
\hline $\begin{array}{l}\text { Utilización de servicios de salud } \\
\text { por los pacientes (dicotómica) }\end{array}$ & RR ajustado $=1,01(0,96$ a 1,15$)$ & $\begin{array}{c}\text { BAJA } \\
\oplus \oplus \ominus \ominus\end{array}$ \\
\hline $\begin{array}{l}\text { Resultados de salud de los pacientes } \\
\text { (dicotómica) }\end{array}$ & RR ajustado $=1,01(0,98$ a 1,04) & $\begin{array}{c}\text { BAJA } \\
\oplus \oplus \ominus \ominus\end{array}$ \\
\hline
\end{tabular}

\section{Conclusiones}

La aplicación de pago por rendimiento en centros de salud de atención ambulatoria puede tener algún efecto en el uso de estu- dios o tratamientos, particularmente en entermedades crónicas,
pero no brindar mejoras en la salud de los pacientes o la utilización de recursos del sistema de salud por parte de los mismos.

\section{Comentario}

Entre los diferentes métodos de pago utilizados en los centros de atención médica ambulatoria resulta interesante analizar principalmente el pago per cápita, por atención brindada y por rendimiento. El primero es aquel en el que el proveedor, en este caso el médico, recibe una tarifa fija por adelantado por cada individuo inscrito con él por un período fijo. Las ventajas de este modelo son su fácil implementación y el incentivo que genera en los médicos a limitar el uso de servicios innecesarios, pero, al mismo tiempo, puede llevar a escatimar el cuidado de los pacientes o elegir en sus capitas pacientes sin comorbilidades que no requieran seguimiento cercano. El pago por atención brindada consiste en un pago retrospectivo que se realiza a cada profesional por prestación realizada. En este caso se evalúa y premia la cantidad de trabajo realizado, sin contemplar la calidad de la atención o el uso correcto o no de los servicios. Por último, el pago por rendimiento es la remuneración del proveedor de salud, ya sean médicos, grupos de personas, o instituciones, según su rendimiento en un tiempo determinado. En este caso se evalúa la calidad de la atención, eficacia y se premia cumplir con ciertos objetivos. Al plantear los diferentes métodos teóricamente se podría pensar que el pago por rendimiento es superior a los demás, al incentivar a los médicos a trabajar de la mejor manera posible, pero llamativamente este trabajo no demuestra tal cuestión. Entre las conclusiones del estudio podemos observar que este modelo puede tener algún efecto en el uso de los recursos, pero a pesar de esto, los pacientes no presentan mejoras en su salud y consultan de igual manera que con los otros tipos de pagos. Esto se podría explicar por la dificultad al intentar implementar el método y el cómo se debe medir el rendimiento profesional, cómo se define un "buen médico".

\section{Conclusiones del comentador}

El pago por rendimiento en los centros de atención ambulatoria puede mejorar la utilización de los recursos por parte de los médicos pero no generar un impacto en la calidad de la atención o en la salud de los pacientes. Sería interesante evaluar diferentes formas de medir rendimiento, observar si existen diferencias si se plantean como objetivos parámetros de laboratorio o del examen físico o medidas indirectas de la calidad de atención como el cuidado longitudinal con un médico de familia o las interconsultas realizadas a especialistas en un tiempo predeterminado.

María Celia Iñurrategui [ Servicio de Medicina Familiar y Comunitaria del Hospital Italiano de Buenos Aires. maria.inurrategui@ hospitalitaliano.org.ar]

Iñurrategui, M. Impacto de los diferentes métodos de pago para centros de atención ambulatoria. Evid Act Pract Ambul. 2018 79. Comentado de: Yuan, B y col. Payment methods for outpatient care facilities. Cochrane Database of Systematic Reviews 2017, Issue 3. Art. No.: CD011153. PMID: 28253540. 